\title{
Doppler flow phantom failure detection by combining empirical mode decomposition and independent component analysis with short time Fourier transform
}

\author{
Giorgia Fiori $^{1}$, Fabio Fuiano ${ }^{1}$, Andrea Scorza ${ }^{1}$, Maurizio Schmid ${ }^{1}$, Silvia Conforto ${ }^{1}$, Salvatore A. Sciuto ${ }^{1}$ \\ ${ }^{1}$ Department of Industrial, Electronic and Mechanical Engineering, ROMA TRE University, Via della Vasca Navale 79, 00146 Rome, Italy
}

\begin{abstract}
Nowadays, objective protocols and criteria for the monitoring of phantoms failures are still lacking in literature, despite their technical limitations. In such a context, the present work aims at providing an improvement of a previously proposed method for the Doppler flow phantom failures detection. Such failures were classified as low frequency oscillations, high velocity pulses and velocity drifts. The novel objective method, named EMoDICA-STFT, is based on the combined application of the Empirical Mode Decomposition (EMD), Independent Component Analysis (ICA) and Short Time Fourier Transform (STFT) techniques on Pulsed Wave (PW) Doppler spectrograms. After a first series of simulations and the determination of adaptive thresholds, phantom failures were detected on real PW spectrograms through the EMoDICA-STFT method. Data were acquired from two flow phantom models set at five flow regimes, through a single ultrasound (US) diagnostic system equipped with a linear, a convex and a phased array probe, as well as with two configuration settings. Despite the promising outcomes, further studies should be carried out on a greater number of Doppler phantoms and US systems as well as including an in-depth investigation of the proposed method uncertainty.
\end{abstract}

\section{Section: RESEARCH PAPER}

Keywords: EMD; ICA; STFT; flow phantom failures; PW Doppler

Citation: Giorgia Fiori, Fabio Fuiano, Andrea Scorza, Maurizio Schmid, Silvia Conforto, Salvatore Andrea Sciuto, Doppler flow phantom failure detection by combining empirical mode decomposition and independent component analysis with short time Fourier transform, Acta IMEKO, vol. 10, no. 4, article 29, December 2021, identifier: IMEKO-ACTA-10 (2021)-04-29

Section Editor: Roberto Montanini, Università di Messina and Alfredo Cigada, Politecnico di Milano, Italy

Received August 2, 2021; In final form December 4, 2021; Published December 2021

Copyright: This is an open-access article distributed under the terms of the Creative Commons Attribution 3.0 License, which permits unrestricted use, distribution, and reproduction in any medium, provided the original author and source are credited.

Corresponding author: Giorgia Fiori, e-mail: giorgia.fiori@uniroma3.it

\section{INTRODUCTION}

Doppler flow phantoms are standard reference test devices usually employed in Quality Controls (QCs) for ultrasound (US) system performance evaluation [1]-[3]. They can simulate the main acoustic characteristics of biological tissues and reproduce repeatable flows whose regimes are similar to those in blood vessels [4]-[7]. To date, the lack of a generally accepted standard for B-mode and Doppler [8]-[12], has led to a limitation on US phantoms use. It should be noticed that, even though such devices are widespread on the market, they are still not included in a shared standard that focuses in detail on periodic and objective checks of their metrological and functional characteristics. This seems to be odd, since the existing commercial Doppler phantoms show several technical limitations [2], [3], [13], [14] affecting their reliability and traceability for Doppler QC testing. The main drawbacks of the most commonly used Doppler phantom model, which is the flow phantom, are the desiccation over time of the Tissue Mimicking Material (TMM), the tendency of Blood Mimicking Fluid (BMF) particles to form agglomerates and/or air bubbles, and the inconsistency of phantom acoustic and pump mechanical properties over time [3]. Despite the awareness of such limitations, objective protocols and criteria for the monitoring of the phantom defects degree are still lacking in literature. In particular, some studies focused their attention on two different kinds of Doppler phantom stability, i.e., TMM and BMF stability [15]-[18]. The former refers to any physical modification in the TMM, while the latter indicates the presence of any solid and/or gaseous element in the BMF. More in detail, TMM stability can be compromised because of (a) TMM fracture e.g., due to desiccation over time, (b) TMM erosion or BMF leakage e.g., due to BMF action through time on TMM in wall-less flow phantoms or tubing material rupture. On the other hand, BMF stability highly depends on (a) any presence of air bubbles, particles 
agglomerates or TMM debris, and (b) unwanted variations of flow velocity regimes. Nevertheless, stability assessment is carried out through a visual qualitative evaluation [15], [16], or without a specific and rigorous protocol [17], [18]. In the existing literature, there are other studies investigating and detecting the failures that could possibly compromise the stability of devices used in both biomedical and mechanical fields. However, there are some issues that nowadays should be taken into account: in [19], for example, it has been pointed out that a specific standard for mechanical heart pumps testing procedures was still missing. Investigations into such issues were limited to the early evaluation of the failures using an analysis technique along with device testing before surgical implantation. On the other hand, in [20] centrifugal pump failures have been reviewed, highlighting the lack of an integrated system able to monitor all the major pump failures.

In such a context, the present study focuses on the improvement and testing of a previously developed Short Time Fourier Transform-based image analysis method [21] for the automatic detection of the main Doppler flow instabilities that may arise. The proposed improved method is based on the application of Empirical Mode Decomposition (EMD) and Independent Component Analysis (ICA) techniques combined with the Short Time Fourier Transform (STFT), namely EMoDICA-STFT, to automatically evaluate the phantom failures through Pulsed Wave (PW) Doppler spectrograms.

EMD is a single-channel technique [22], [23], firstly introduced in [24], to obtain the decomposition of a signal in time. It is widely used in combination with ICA for the effective processing of electrophysiological signals [25], [26]. An interesting feature of such a combination is the possibility of successfully extracting both oscillatory and spike-like sources [22].

STFT is a time-frequency spectral analysis technique, widely used in several scientific fields, such as structural mechanics, aeronautics, and biomedical engineering. It has been applied in structural health monitoring fields, to detect damages in existing structures [27], to classify and predict delamination in smart composite laminates [28], to reveal corrosion and fatigue cracks in aircraft structures [29], and to analyse physiological signal characteristics and determine relevant parameters [30]-[33].

The goal of the present work is the implementation and testing of the EMoDICA-STFT method: it processes PW Doppler spectrograms collected from two different Doppler flow phantoms through a single intermediate technology level US system equipped with three array probes (linear, convex, and phased array) at their central Doppler frequency.

In Section 2, a brief overview of the techniques adopted in the proposed method is provided, and their combined application on three simulation cases is described. In Section 3, the experimental setup used in this study and the EMoDICASTFT method application to PW spectrograms is discussed. In Section 4 results are presented and discussed on the basis of infra- and inter-phantom differences in the detected failures. Finally, in the concluding section, the major achievements and future developments of the research hereby presented will be reported.

\section{EMODICA-STFT METHOD APPLICATION TO PHANTOM FAILURES DETECTION}

BMF instability sources can be identified as any presence of air bubbles, particle agglomerates or TMM debris, and unwanted variations of flow velocity regimes. Consequently, the present study focuses on their detection in PW spectrograms, with particular reference to the following phantom failures:

1. low frequency oscillations caused by any pump or hydraulic dampener inability to deliver a constant flow velocity in correspondence of a continuous flow regime setting;

2. high velocity pulses caused by any particle agglomerates or TMM debris in the phantom flow;

3. flow velocity drifts due to the unwanted onset of the pump acceleration (e.g., deriving from a failure in the control system).

\subsection{EMD, ICA and STFT techniques}

Empirical Mode Decomposition [22], [24] is a signalprocessing tool that, through an iterative process, decomposes a signal $x(t)$ into a finite set of Intrinsic Mode Functions (IMFs) and a residual $R_{M}(t)$, as follows:

$$
x(t)=\sum_{i=1}^{M} I M F_{i}(t)+R_{M}(t),
$$

where $I M F_{i}(t)$ is the i-th oscillatory mode. Each IMFs has the following properties [22]: (a) it contains one frequency only, which is referred to as the instantaneous frequency, (b) its frequency is different from all the ones of the other functions, (c) it has zero mean value, and (d) it is an oscillatory function. One of the main advantages of EMD, as compared to other decomposition techniques [22], is that it does not require any apriori knowledge of the signal to be decomposed.

In turn, Independent Component Analysis is a blind source separation technique [34], applied to a set of recorded signals $y_{i}(t)$ whose aim is the extraction of unknown sources $s_{i}(t)$, named Independent Components (ICs), under the assumption of statistical independency. In particular, $y_{i}(t)$ can be expressed as a linear combination of $s_{i}(t)$, as follows:

$$
\vec{y}(t)=[A] \cdot \vec{s}(t),
$$

where $[A]$ is an unknown matrix, called mixing matrix. In the present study, a computationally improved ICA method was applied, namely FastICA [35], which was implemented in MATLAB environment as a software package [36].

Finally, Short Time Fourier Transform is a set of Fourier transforms performed on a signal, which is subdivided into overlapped or non-overlapped temporal segments, through a translating window (e.g., rectangular, Hanning) in time. The Fourier transform is applied under the hypothesis of pseudostationarity of the temporal segments [37], which is achieved through the choice of a short translating window. The STFT expression for a generic discrete signal $\chi(n)$, is the following:

$$
\operatorname{STFT}(n, \omega)=\sum_{h=-\infty}^{+\infty} x(n+h) w_{N}(h) e^{-j \omega h},
$$

where $w_{N}(n)$ is the translating window. The corresponding normalized, real-valued, non-negative spectrogram $S_{x}(n, \omega)$ can be computed through the following expression:

$$
S_{x}(n, \omega)=\frac{2}{N} \cdot C F \cdot|\operatorname{STFT}(n, \omega)|^{2},
$$

where $N$ is the sample window length and $C F$ is a correction factor varying according to the chosen window amplitude [21]. Therefore, (4) has the advantage of taking the applied window type into account. 


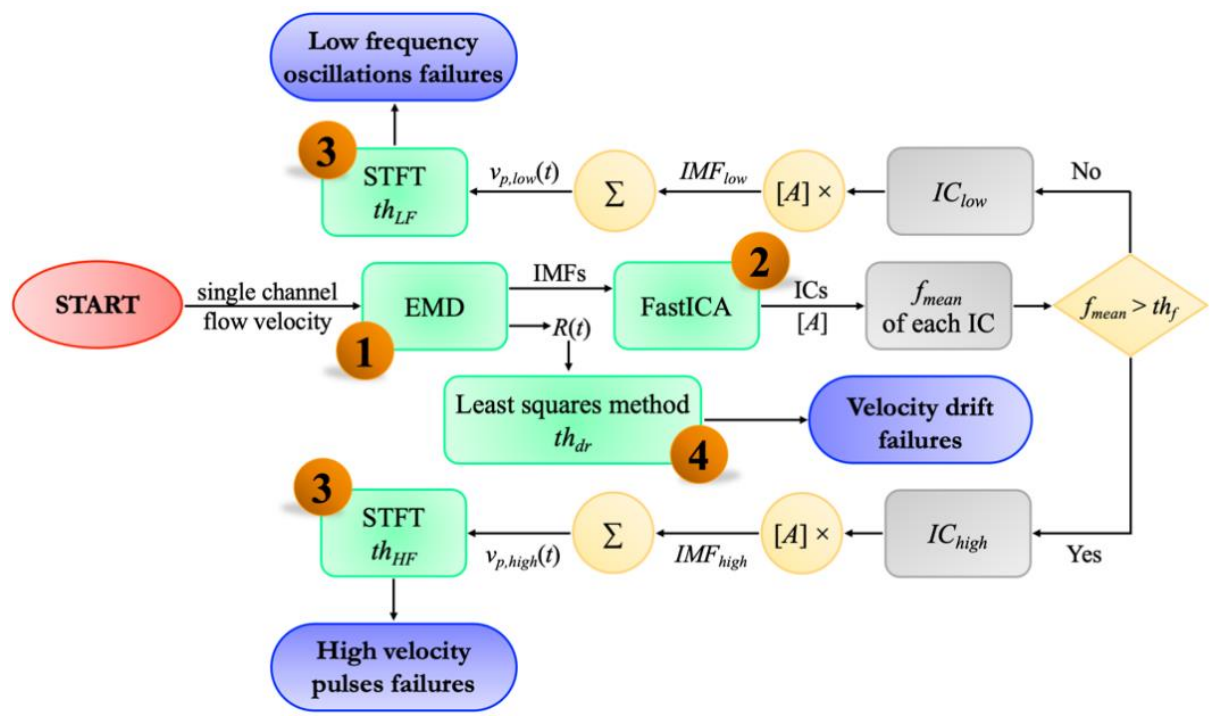

Figure 1. Block diagram of the proposed EMoDICA-STFT method for flow phantom failures detection.

\subsection{EMoDICA-STFT simulated application}

This subsection describes the EMoDICA-STFT method proposed to assess the phantom failures, through the combination of the previously described techniques, as shown in the block diagram (Figure 1). The following steps were applied to a real flow maximum velocity signal $v_{p}(t)$ lasting $\sim 60 \mathrm{~s}$, which was derived from PW spectrograms according to the procedure which will be described in Section 3.2. It was chosen among the available data because it is a representative case study: in fact, the signal shows both high-velocity pulses and low-frequency oscillations. A simulated velocity drift $v_{d r}(t)$ of $0.06 \mathrm{~cm} \cdot \mathrm{s}^{-2}$ was added to $v_{p}(t)$ as an increasing trend in time, obtaining a velocity signal $v_{p, t o t}(t)$ (Figure 2), and then, the steps described in the following and represented in Figure 1, were applied.

First step. The first step is the application of EMD to $v_{p, t o t}(t)$ : the IMFs and the residual $R(t)$ are retrieved on the basis of (1).

Second step. The second step is the application of FastICA to the IMFs in order to compute the ICs and the mixing matrix $[A]$. At this point, the mean frequency $f_{\text {mean }}$ of each independent component is obtained, and a frequency threshold $t h_{f}=0.5 \mathrm{~Hz}$ is selected to discriminate between high- and low-frequency content ICs. Therefore, two different groups of ICs are obtained, namely $I C_{\text {low }}$ and $I C_{\text {bigh }}$. The latter are multiplied for the mixing matrix $[A]$ according to (2), to back-reconstruct the corresponding oscillatory modes $I M F_{l o w}$ and $I M F_{\text {high }}$. Finally, the modes of each group are summed together to reconstruct two signals $v_{p, l o w}(t)$ and $v_{p, b i g h}(t)$ derived from the signal $v_{p, t o t}(t)$, where the

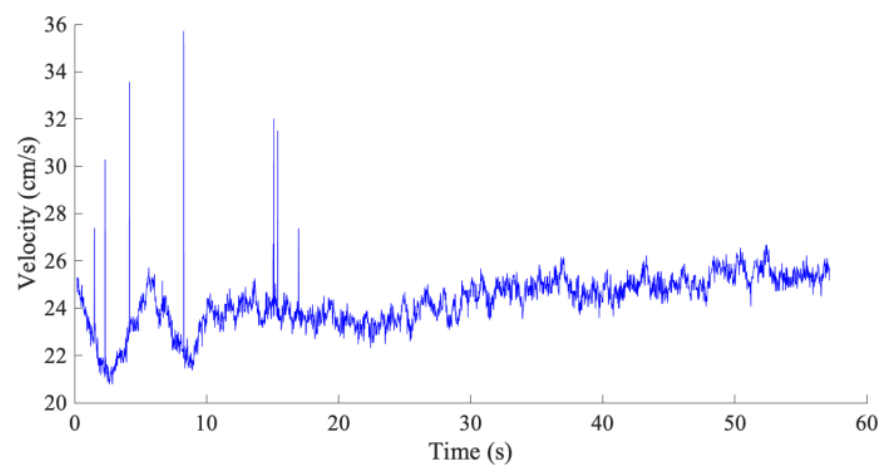

Figure 2. Real flow maximum velocity signal with low frequency oscillations, high velocity pulses with a $0.06 \mathrm{~cm} \cdot \mathrm{s}^{-2}$ velocity drift. first one has frequency contents lower than $t_{f}$ (Figure 3 a), while the second one has frequency contents higher than $t b_{f}$ (Figure 3 b).

Third step. The third step is the STFT application, according to (3) and (4), to $v_{p, l o n}(t)$ and $v_{p, b i g h}(t)$, with the settings reported in Table 1. As already done in [21], the spectral window chosen is the Hanning window, whose expression is the following:

$$
w_{N}(n)=\frac{1}{2}\left(1-\cos \frac{2 \pi n}{N}\right) .
$$

After the STFT application, two mesh plots are obtained. In this way, it is possible to carry out the failure detection by distinguishing the contributions of the low frequency oscillations and high velocity pulses in the spectrograms of $v_{p, l o w}(t)$ and $v_{p, b i g h}(t)$, respectively.

Low frequency oscillations are represented in the mesh plot of $v_{p, l o n}(t)$ normalized spectrogram as frequency pulses (Figure 4 $\mathrm{a}, \mathrm{b})$. Therefore, the detection of an oscillation occurs when $S_{x}$
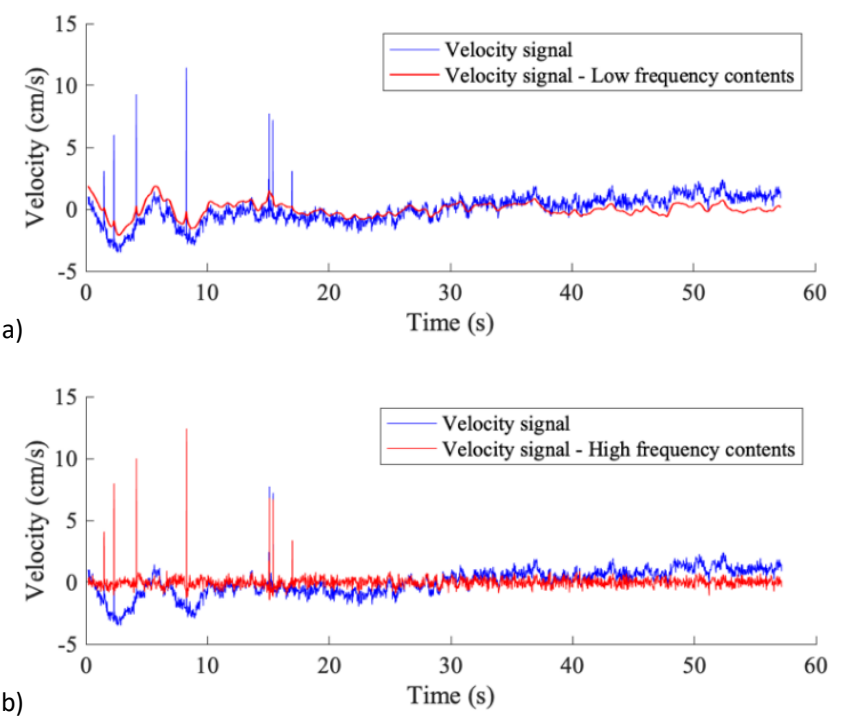

Figure 3. (a) Reconstructed signal $v_{p, \text { low }}(t)$ with low frequency contents superimposed to $v_{p, t o t}(t)$ after the offset removal; (b) reconstructed signal $v_{p, \text { high }}(t)$ with high frequency contents superimposed to $v_{p, t o t}(t)$ after the offset removal. 
Table 1. STFT parameters setting.

\begin{tabular}{|c|c|c|}
\hline Parameter & Symbol & Value \\
\hline Sampling frequency $(\mathrm{Hz})$ & $f_{s}$ & 100 \\
\hline Spectral window & \multicolumn{2}{|c|}{ Hanning } \\
\hline Window length (samples) & $N$ & 100 \\
\hline Overlap (samples) & $N_{\text {overlap }}$ & 60 \\
\hline Zero-padding (samples) & $N_{\text {zero-pad }}$ & 50 \\
\hline Correction factor & $C F$ & 2 \\
\hline Temporal resolution (s) & $\Delta t$ & 0.4 \\
\hline Frequency resolution $(\mathrm{Hz})$ & $\Delta f$ & $\sim 0.7$ \\
\hline
\end{tabular}

shows a pulse both limited in time (according to the oscillation period) and frequency $(\sim 0 \mathrm{~Hz})$, whose amplitude is higher than the threshold $t h_{L F}$, automatically determined as follows:

$$
t h_{L F}=\frac{\sigma_{v}^{2}}{\Delta f} \cdot G,
$$

where $\sigma_{v}$ is the flow velocity standard deviation depending on the phantom model, $\Delta f$ is the STFT frequency resolution, and $G$ is a safety factor that in this study was chosen equal to 10 .

In turn, high velocity pulses are represented, as shown in the normalized spectrogram of $v_{p, b i g h}(t)$, by a window covering almost all the frequency components in the mesh plot (Figure $4 \mathrm{c}, \mathrm{d}$ ). Therefore, the detection of a pulse occurs when the average amplitude of the frequency components, between 5 and $30 \mathrm{~Hz}$, related to a single temporal instant is higher than the threshold $t h_{H F}$. The latter can be automatically determined, by considering the sampling frequency $f_{s}$ of $v_{p}(t)$, as follows:

$$
t h_{H F}=\frac{\sigma_{v}^{2}}{\Delta f} \cdot G \cdot \frac{F_{f r}}{f_{s} / 2},
$$

where $F_{f r}$ is a factor that considers the entity of the frequency range in which the failure occurs in the normalized spectrogram. In this case, where a frequency range between 5 and $30 \mathrm{~Hz}$ was considered, $F_{f r}=25 \mathrm{~Hz}$. The choice to reduce the frequency range in which the detection is carried out was necessary to compensate for non-ideal pulses [21].

Fourth step. The fourth step of the EMoDICA-STFT method is the detection of the velocity drifts from the EMD residual $R(t)$. After the application of the least squares method to $R(t)$ (Figure 5 ), it is possible to evaluate any velocity drift through the computation of the angular coefficient $m$ of the straight line that best approximates the residual trend. In particular, the detection of a velocity drift occurs when $|m|$ is higher than the threshold $t h_{d r}$, that can be automatically determined as:

$$
t h_{d r}=\frac{\sigma_{v}}{t_{P W}} \cdot G,
$$

where $t_{P W}$ is the velocity signal duration, while the safety factor $G$ for the velocity drift detection was chosen equal to 2 . The advantage of (8) relies on its dependence on the phantom flow velocity standard deviation $\sigma_{v}$ so that the velocity drift perception is not affected by human eye subjectivity. The retrieved angular coefficient of $R(t)$, shown in Figure 5, is lower than the simulated velocity drift added to $v_{p}(t)$. This is likely due to the combination of $v_{d r}(t)$ with the pre-existing trend of the real velocity signal under analysis.
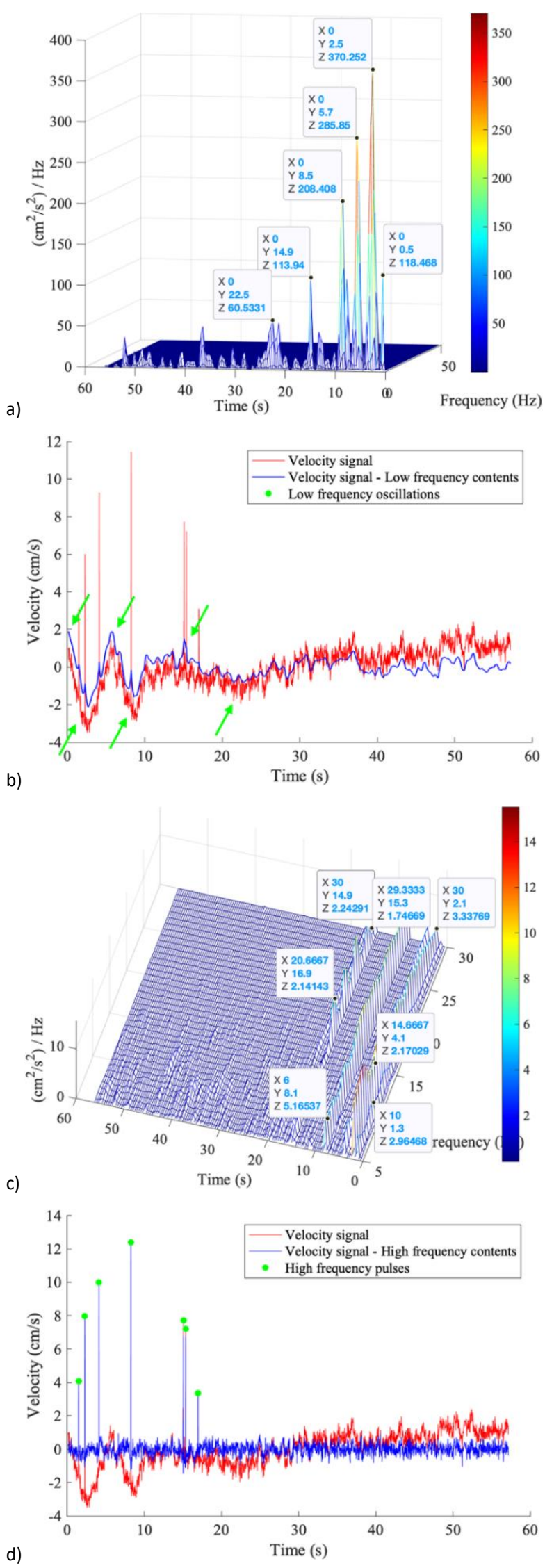

Figure 4. Low frequency contents signal $v_{p \text {, low }}(t)$ represented through (a) a mesh plot with the detected frequency peaks $\left(t_{L F}=60 \mathrm{~cm}^{2} \cdot \mathrm{s}^{-2} \cdot \mathrm{Hz}^{-1}\right)$ and $(\mathrm{b})$ its temporal evolution together with the signal $v_{p, t o t}(t)$; High frequency contents signal $v_{p, \text { high }}(t)$ represented through (c) a mesh plot with the detected frequency windows $\left(\right.$ th $\left.h_{H F}=30 \mathrm{~cm}^{2} \cdot \mathrm{s}^{-2} \cdot \mathrm{Hz}^{-1}\right)$ and (d) its temporal evolution together with the signal $v_{p, \text { tot }}(t)$. 


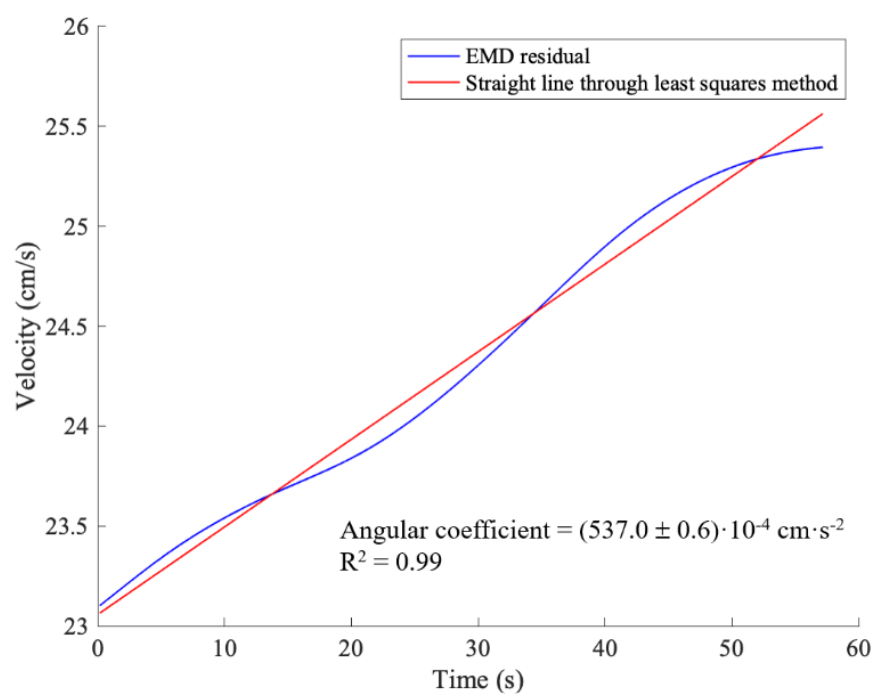

Figure 5. Least squares method applied to the EMD residual in the case of a $0.06 \mathrm{~cm} \cdot \mathrm{s}^{-2}$ velocity drift added to the real maximum velocity signal $v_{p}(t)$.

Finally, this simulation was repeated in two further cases to test the proposed method for different starting conditions: (a) $v_{p}(t)$ with an additional velocity drift of $0.03 \mathrm{~cm} \cdot \mathrm{s}^{-2}$ and (b) $v_{p}(t)$ with an additional velocity drift of $0.09 \mathrm{~cm} \cdot \mathrm{s}^{-2}$. The EMoDICASTFT method identified the same low frequency oscillations and high velocity pulses retrieved for the first simulation, while the obtained velocity drift angular coefficients were $(164.1 \pm 0.4) \cdot 10$ ${ }^{4} \mathrm{~cm} \cdot \mathrm{s}^{-2}\left(\mathrm{R}^{2}=0.97\right)$ and $(684.9 \pm 0.7) \cdot 10^{-4} \mathrm{~cm} \cdot \mathrm{s}^{-2}\left(\mathrm{R}^{2}=0.99\right)$ for case (a) and (b), respectively.

\section{MATERIALS AND EXPERIMENTAL DATA}

In the present study, the EMoDICA-STFT method for the phantom failures assessment, implemented in MATLAB, is proposed as an improvement of a procedure previously described in [21]. This is achieved through the experimental setup described in the following section.

\subsection{Experimental setup}

Data were collected by using two Doppler flow phantoms, whose main characteristics are reported in Table 2. The first phantom under test (PUT) is Gammex, Optimizer ${ }^{\circledR} 1425 \mathrm{~A}$, a self-contained device [38] able to provide constant or pulsatile flow in the $1.7-12.5 \mathrm{ml} \cdot \mathrm{s}^{-1}$ range, through an electric flow controller. The second PUT is CIRS, model 069, an easy-to-

Table 2. Main characteristics of the two Doppler flow phantoms [38], [39].

\begin{tabular}{lll}
\hline \multicolumn{1}{c}{ Parameter } & \multicolumn{1}{c}{\begin{tabular}{c}
\multicolumn{1}{c}{ Gammex } \\
Optimazer
\end{tabular}} & \multicolumn{1}{c}{ 1425A }
\end{tabular}

(*) The flow velocity standard deviations were estimated from the specifications reported in the phantoms datasheets.
Table 3. Doppler phantoms flow rate and mean flow velocity settings.

\begin{tabular}{llcc}
\hline $\begin{array}{c}\text { Flow } \\
\text { phantom }\end{array}$ & \multicolumn{1}{c}{ Flow regime } & $\begin{array}{c}\text { Flow rate } \\
\boldsymbol{Q}\left(\mathbf{m l} \cdot \mathbf{s}^{-1}\right)\end{array}$ & $\begin{array}{c}\text { Mean flow velocity } \\
\boldsymbol{v}\left(\mathbf{c m} \cdot \mathbf{s}^{-1}\right)\end{array}$ \\
\hline \multirow{2}{*}{ Gammex } & Low - L & 2.6 & 13.2 \\
Optimazer ${ }^{\circledR}$ & Low-medium - LM $_{\mathrm{f}}$ & 3.7 & 18.8 \\
1425A & Medium - $\mathrm{M}_{\mathrm{f}}$ & 4.8 & 24.4 \\
& Medium-high - $\mathrm{MH}_{\mathrm{f}}$ & 5.9 & 30.0 \\
& High - $\mathrm{H}_{\mathrm{f}}$ & 7.0 & 35.7 \\
& Low - L & 2.4 & 13.3 \\
CIRS & Low-medium - $\mathrm{LM}_{\mathrm{f}}$ & 3.4 & 18.8 \\
model 069 & Medium - $\mathrm{M}_{\mathrm{f}}$ & 4.4 & 24.3 \\
& Medium-high - $\mathrm{MH}_{\mathrm{f}}$ & 5.4 & 29.8 \\
& High - $\mathrm{H}_{\mathrm{f}}$ & 6.4 & 35.4 \\
\hline
\end{tabular}

assemble simulator [39], able to provide an average flow velocity between 5 and $68 \mathrm{~cm} \cdot \mathrm{s}^{-1}$, through the action of a peristaltic pump, providing a pulsatile flow. The latter can be converted into a constant flow through the connection of a dampener.

The acquisition started after 2 hours of phantom warm-up. In order to test their stability, five constant flow rate values (low $\mathrm{L}_{\mathrm{f}}$, low-medium $\mathrm{LM}_{\mathrm{f}}$, medium $\mathrm{M}_{\mathrm{f}}$, medium-high $\mathrm{MH}_{\mathrm{f}}$ and high $\mathrm{H}_{\mathrm{f}}$ ) were set, as shown in Table 3. Doppler phantom characteristics are not consistent, therefore, flow values were differently set to guarantee the same mean velocity regimes $\left(v_{\text {Gammex }}=v_{\text {CIRS }}\right)$, as detailed in [21].

A single US system equipped with three US probes (linear, convex, and phased array) was used to acquire six PW spectrograms lasting $\sim 10 \mathrm{~s}$ for each flow regime. Data were collected with two different PW Doppler settings, namely set A and set $\mathrm{B}$, reported in Table 4 , in order to make a comparison of the stability performance between the two test objects under different setting conditions. The sample volume length was maintained fixed for both phantoms and settings, whereas the sample volume depth was changed according to the phantom model attenuation, and kept consistent for set A and set B. As regards the insonification angle, it was varied according both to the probe positioning on the scanning surface and to the different tube slopes of the two phantoms.

Table 4. PW Doppler main configuration settings.

\begin{tabular}{|c|c|c|c|c|c|}
\hline \multirow{3}{*}{\begin{tabular}{l}
\multicolumn{1}{c}{ Parameter } \\
Doppler \\
frequency $(\mathrm{MHz})$
\end{tabular}} & \multicolumn{3}{|c|}{ Set $A$} & \multicolumn{2}{|c|}{ Set B } \\
\hline & \multirow[t]{2}{*}{ Gammex } & CIRS & \multicolumn{2}{|c|}{ Gammex } & \multirow[t]{2}{*}{ CIRS } \\
\hline & & $L=5.21$ & $C=2.50$ & $\mathrm{P}=$ & \\
\hline Wall filter & \multicolumn{3}{|c|}{ Minimum } & \multicolumn{2}{|c|}{ Medium } \\
\hline $\begin{array}{l}\text { Spectrum } \\
\text { resolution }\end{array}$ & \multicolumn{2}{|c|}{ Minimum } & & \multicolumn{2}{|c|}{$\begin{array}{c}\mathrm{L}=\text { Medium } \\
\mathrm{C}, \mathrm{P}=\text { Minimum }\end{array}$} \\
\hline $\begin{array}{l}\text { Sample volume } \\
\text { length }(\mathrm{cm})\end{array}$ & \multicolumn{5}{|c|}{3.0} \\
\hline $\begin{array}{l}\text { Sample volume } \\
\text { depth }(\mathrm{mm})\end{array}$ & 48 & 40 & \multicolumn{2}{|c|}{48} & 40 \\
\hline $\begin{array}{l}\text { Insonification } \\
\text { angle }\left({ }^{\circ}\right)\end{array}$ & 52 & $\begin{array}{l}\mathrm{L}, \mathrm{C}= \\
\mathrm{P}=5\end{array}$ & & 52 & $\begin{array}{c}L, C=70 \\
P=55\end{array}$ \\
\hline $\begin{array}{l}\text { PW spectrogram } \\
\text { duration (s) }\end{array}$ & \multicolumn{5}{|c|}{$\sim 10$} \\
\hline $\begin{array}{l}\text { PW spectrogram } \\
\text { total duration (s) }\end{array}$ & \multicolumn{5}{|c|}{$\sim 60$} \\
\hline
\end{tabular}

$\mathrm{L}=$ linear, $\mathrm{C}=$ phased, $\mathrm{P}=$ phased array probe. 


\subsection{EMODICA-STFT on PW spectrograms}

Each PW spectrogram was processed for the detection of the maximum velocity waveform, as in [21]. Pixel coordinates $p x_{\max }$ associated to the maximum velocity values were detected through a gray level adaptive threshold th max $_{\text {ax }}$ automatically determined as $10 \%$ of the maximum gray level value [40]. At this point, $p x_{\max }$ were associated to the corresponding flow velocity values $v_{\max }$ for each temporal instant, taking into consideration the maximum value displayed on the PW velocity scale. Then, the six $v_{\max }$ signals obtained for the same flow regime were juxtaposed. The EMoDICA-STFT application was implemented according to the block diagram in Figure 1 and with the STFT settings reported in Table 1 . The thresholds applied for the detection of failures show different values between the Doppler phantoms under test because of the different flow velocity standard deviation (Table 2). According to (6)-(8), the computed threshold values were: $t h_{L F}=60 \mathrm{~cm}^{2} \cdot \mathrm{s}^{-2} \cdot \mathrm{Hz}^{-1}, t h_{H F}=30 \mathrm{~cm}^{2}$. $\mathrm{s}^{-2} \cdot \mathrm{Hz}^{-1}$ and $t h_{d r}=7 \cdot 10^{-2} \mathrm{~cm} \cdot \mathrm{s}^{-2}$, for Gammex $1425 \mathrm{~A}$ and $t h_{L F}=$ $135 \mathrm{~cm}^{2} \cdot \mathrm{s}^{-2} \cdot \mathrm{Hz}^{-1}, t h_{H F}=67 \mathrm{~cm}^{2} \cdot \mathrm{s}^{-2} \cdot \mathrm{Hz}^{-1}$ and $t h_{d r}=10 \cdot 10^{-2} \mathrm{~cm} \cdot$ $\mathrm{s}^{-2}$, for CIRS model 069. It is worth noting that, due to the threshold dependency on flow standard deviation, the higher threshold values retrieved for CIRS phantom are a first indicator of its lower performance as compared to the Gammex phantom.

\section{RESULTS AND DISCUSSION}

The number of phantom failures detected for the two test objects according to the US probe, the flow regime $\left(\mathrm{L}_{\mathrm{f}}, \mathrm{LM}_{\mathrm{f}}, \mathrm{M}_{\mathrm{f}}\right.$, $\mathrm{MH}_{\mathrm{f}}$ and $\mathrm{H}_{\mathrm{f}}$ ) and the PW Doppler settings (set A and set $\mathrm{B}$ ) is reported in Table 5 and Table 6 . According to [41], the standard deviation values can be computed as the square root of the counted value. The EMoDICA-STFT method did not detect any velocity drift failure on the two phantoms, because the angular coefficients $|m|$ of the straight lines, obtained by applying the least squares method to all the EMD residuals, were always lower than the determined $t h_{d r}$.

As regards the Gammex 1425A phantom, it should be noted that, independently from the US probe considered, the number of low frequency oscillations is globally limited, except for the $\mathrm{LM}_{\mathrm{f}}$ flow regime, in set A and set B. As shown in Figure 6, a sinusoidal trend is clearly visible, therefore suggesting that the phantom electric flow controller seems no longer able to guarantee a constant flow regime of $3.7 \mathrm{ml} \cdot \mathrm{s}^{-1}$. On the other hand, both convex and phased array probes show a higher number of high velocity pulses with respect to the linear array probe, suggesting a probe-dependent sensitivity to BMF particle agglomerates. Furthermore, independently from the probe, the low flow regime $\mathrm{L}_{\mathrm{f}}$ shows the highest number of high velocity pulses. This may be due to the fact that the flow velocity is too low to dissolve the particle agglomerates.

Table 5. Number of detected failures according to the US probe, the flow regime and PW Doppler settings for Gammex $1425 \mathrm{~A}$.

\begin{tabular}{|c|c|c|c|c|c|c|c|}
\hline \multirow{2}{*}{ US probe } & \multirow{2}{*}{$\begin{array}{l}\text { Flow } \\
\text { regime }\end{array}$} & \multirow{2}{*}{$\begin{array}{l}\text { PW Doppler } \\
\text { setting }\end{array}$} & \multirow{2}{*}{$\begin{array}{l}\text { Low frequency oscillation } \\
\qquad h_{L F}=60 \mathrm{~cm}^{2} \cdot \mathrm{s}^{-2} \cdot \mathrm{Hz}^{-1}\end{array}$} & \multirow{2}{*}{$\begin{array}{l}\text { High velocity pulse } \\
t h_{H F}=30 \mathrm{~cm}^{2} \cdot \mathrm{s}^{-2} \cdot \mathrm{Hz}^{-1}\end{array}$} & \multicolumn{3}{|c|}{ Velocity drift } \\
\hline & & & & & $t h_{d r}=7 \cdot 10^{-2} \mathrm{~cm} \cdot \mathrm{s}^{-2}$ & $\begin{array}{l}\text { Angular coefficient } \\
\qquad m\left(\mathrm{~cm} \cdot \mathrm{s}^{-2}\right)\end{array}$ & $\mathbf{R}^{2}$ \\
\hline \multirow{10}{*}{ Linear } & \multirow{2}{*}{$\mathrm{L}_{f}$} & set $A$ & $6 \pm 2$ & $7 \pm 3$ & - & $-0.9 \cdot 10^{-2}$ & 0.99 \\
\hline & & set $B$ & - & $3 \pm 2$ & - & $-1.0 \cdot 10^{-2}$ & 0.94 \\
\hline & \multirow[b]{2}{*}{$\mathrm{LM}_{\mathrm{f}}$} & set $A$ & $49 \pm 7$ & - & - & $-0.9 \cdot 10^{-2}$ & 0.99 \\
\hline & & set $B$ & $45 \pm 7$ & - & - & $2.1 \cdot 10^{-4}$ & 0.99 \\
\hline & \multirow{2}{*}{$M_{f}$} & set $A$ & - & - & - & $-3.7 \cdot 10^{-3}$ & 0.99 \\
\hline & & set $B$ & - & - & - & $-2.3 \cdot 10^{-3}$ & 0.98 \\
\hline & \multirow{2}{*}{$\mathrm{MH}_{\mathrm{f}}$} & set $A$ & - & - & - & $4.0 \cdot 10^{-3}$ & 0.99 \\
\hline & & set $B$ & - & $8 \pm 3$ & - & $-3.8 \cdot 10^{-3}$ & 0.89 \\
\hline & \multirow[b]{2}{*}{$\mathrm{H}_{\mathrm{f}}$} & set $A$ & - & - & - & $-0.9 \cdot 10^{-2}$ & 0.98 \\
\hline & & set $B$ & - & $1 \pm 1$ & - & $2.8 \cdot 10^{-3}$ & 0.99 \\
\hline \multirow{10}{*}{ Convex } & \multirow{2}{*}{$\mathrm{L}_{f}$} & set $A$ & $2 \pm 1$ & $44 \pm 7$ & - & $4.0 \cdot 10^{-2}$ & 0.98 \\
\hline & & set $B$ & - & $14 \pm 4$ & - & $-4.3 \cdot 10^{-4}$ & 0.98 \\
\hline & \multirow{2}{*}{$\mathrm{LM}_{\mathrm{f}}$} & set $A$ & $47 \pm 7$ & $2 \pm 1$ & - & $-4.9 \cdot 10^{-3}$ & 0.99 \\
\hline & & set $B$ & $44 \pm 7$ & $2 \pm 1$ & - & $-1.2 \cdot 10^{-2}$ & 0.99 \\
\hline & \multirow[b]{2}{*}{$M_{f}$} & set $A$ & - & $9 \pm 3$ & - & $-1.6 \cdot 10^{-3}$ & 0.99 \\
\hline & & set $B$ & - & $1 \pm 1$ & - & $-2.4 \cdot 10^{-3}$ & 0.99 \\
\hline & \multirow[b]{2}{*}{$\mathrm{MH}_{\mathrm{f}}$} & set $A$ & - & $6 \pm 2$ & - & $-3.3 \cdot 10^{-3}$ & 0.94 \\
\hline & & set $B$ & - & $3 \pm 2$ & - & $-0.9 \cdot 10^{-2}$ & 0.99 \\
\hline & \multirow{2}{*}{$\mathrm{H}_{\mathrm{f}}$} & set $A$ & $2 \pm 1$ & $2 \pm 1$ & - & $-0.6 \cdot 10^{-2}$ & 0.98 \\
\hline & & set $B$ & - & $1 \pm 1$ & - & $1.9 \cdot 10^{-5}$ & 0.99 \\
\hline \multirow{10}{*}{ Phased } & \multirow{2}{*}{$\mathrm{L}_{f}$} & set $A$ & - & $25 \pm 5$ & - & $-0.8 \cdot 10^{-3}$ & 0.99 \\
\hline & & set $B$ & - & $4 \pm 2$ & - & $3.1 \cdot 10^{-3}$ & 0.92 \\
\hline & \multirow[b]{2}{*}{$\mathrm{LM}_{\mathrm{f}}$} & set $A$ & $44 \pm 7$ & - & - & $-1.9 \cdot 10^{-2}$ & 0.98 \\
\hline & & set $B$ & $47 \pm 7$ & $4 \pm 2$ & - & $1.2 \cdot 10^{-3}$ & 0.99 \\
\hline & \multirow{2}{*}{$\mathrm{M}_{\mathrm{f}}$} & set $A$ & - & $3 \pm 2$ & - & $-1.4 \cdot 10^{-2}$ & 0.94 \\
\hline & & set $B$ & - & $8 \pm 3$ & - & $1.5 \cdot 10^{-3}$ & 0.96 \\
\hline & \multirow{2}{*}{$\mathrm{MH}_{\mathrm{f}}$} & set $A$ & - & $9 \pm 3$ & - & $1.0 \cdot 10^{-3}$ & 0.96 \\
\hline & & set $B$ & - & $10 \pm 3$ & - & $4.0 \cdot 10^{-3}$ & 0.98 \\
\hline & \multirow{2}{*}{$\mathrm{H}_{\mathrm{f}}$} & set $A$ & - & $6 \pm 2$ & - & $-3.3 \cdot 10^{-3}$ & 0.99 \\
\hline & & set $B$ & - & $5 \pm 2$ & - & $-3.3 \cdot 10^{-3}$ & 0.99 \\
\hline
\end{tabular}


Table 6. Number of detected failures according to the US probe, the flow regime and PW Doppler settings for CIRS model 069.

\begin{tabular}{|c|c|c|c|c|c|c|c|}
\hline \multirow[b]{2}{*}{ US probe } & \multirow{2}{*}{$\begin{array}{l}\text { Flow } \\
\text { regime }\end{array}$} & \multirow{2}{*}{$\begin{array}{l}\text { PW Doppler } \\
\text { setting }\end{array}$} & \multirow{2}{*}{$\begin{array}{l}\text { Low frequency oscillation } \\
\qquad h_{L F}=135 \mathrm{~cm}^{2} \cdot \mathrm{s}^{-2} \cdot \mathrm{Hz}^{-1}\end{array}$} & \multirow{2}{*}{$\begin{array}{l}\text { High velocity pulse } \\
t h_{H F}=67 \mathrm{~cm}^{2} \cdot \mathrm{s}^{-2} \cdot \mathrm{Hz}^{-1}\end{array}$} & \multicolumn{3}{|c|}{ Velocity drift } \\
\hline & & & & & $t h_{d r}=10 \cdot 10^{-2} \mathrm{~cm} \cdot \mathrm{s}^{-2}$ & $\begin{array}{c}\text { Angular coefficient } \\
\qquad m\left(\mathrm{~cm} \cdot \mathrm{s}^{-2}\right)\end{array}$ & $\mathbf{R}^{2}$ \\
\hline \multirow{10}{*}{ Linear } & \multirow{2}{*}{$\mathrm{L}_{f}$} & set $A$ & $4 \pm 2$ & - & - & $3.7 \cdot 10^{-2}$ & 0.99 \\
\hline & & set $B$ & $2 \pm 1$ & - & - & $-0.8 \cdot 10^{-2}$ & 0.99 \\
\hline & \multirow[b]{2}{*}{$\mathrm{LM}_{\mathrm{f}}$} & set $A$ & - & - & - & $3.6 \cdot 10^{-3}$ & 0.95 \\
\hline & & set $B$ & - & - & - & $-1.8 \cdot 10^{-4}$ & 0.99 \\
\hline & \multirow[b]{2}{*}{$M_{f}$} & set $A$ & - & - & - & $-1.9 \cdot 10^{-3}$ & 0.97 \\
\hline & & set $B$ & - & - & - & $-1.0 \cdot 10^{-3}$ & 0.99 \\
\hline & \multirow{2}{*}{$\mathrm{MH}_{\mathrm{f}}$} & set $A$ & $2 \pm 1$ & - & - & $0.8 \cdot 10^{-2}$ & 0.94 \\
\hline & & set $B$ & $11 \pm 3$ & $1 \pm 1$ & - & $2.0 \cdot 10^{-3}$ & 0.99 \\
\hline & \multirow[b]{2}{*}{$\mathrm{H}_{\mathrm{f}}$} & set $A$ & - & - & - & $3.8 \cdot 10^{-4}$ & 0.99 \\
\hline & & set B & $2 \pm 1$ & - & - & $2.3 \cdot 10^{-2}$ & 0.99 \\
\hline \multirow{10}{*}{ Convex } & \multirow[b]{2}{*}{$\mathrm{L}_{f}$} & set $A$ & - & $5 \pm 3$ & - & $2.2 \cdot 10^{-2}$ & 0.99 \\
\hline & & set $B$ & - & $7 \pm 3$ & - & $-4.6 \cdot 10^{-3}$ & 0.99 \\
\hline & \multirow{2}{*}{$\mathrm{LM}_{\mathrm{f}}$} & set $A$ & - & $9 \pm 3$ & - & $-2.0 \cdot 10^{-2}$ & 0.95 \\
\hline & & set $B$ & - & $6 \pm 3$ & - & $1.0 \cdot 10^{-2}$ & 0.93 \\
\hline & \multirow[b]{2}{*}{$M_{f}$} & set $A$ & $1 \pm 1$ & $17 \pm 4$ & - & $2.2 \cdot 10^{-2}$ & 0.95 \\
\hline & & set $B$ & - & $11 \pm 3$ & - & $4.3 \cdot 10^{-3}$ & 0.96 \\
\hline & \multirow{2}{*}{$\mathrm{MH}_{\mathrm{f}}$} & set $A$ & - & $1 \pm 1$ & - & $-1.9 \cdot 10^{-2}$ & 0.99 \\
\hline & & set $B$ & $18 \pm 4$ & $2 \pm 1$ & - & $1.9 \cdot 10^{-2}$ & 0.99 \\
\hline & \multirow{2}{*}{$\mathrm{H}_{\mathrm{f}}$} & set $A$ & $5 \pm 2$ & $2 \pm 1$ & - & $-2.6 \cdot 10^{-2}$ & 0.99 \\
\hline & & set B & $1 \pm 1$ & $1 \pm 1$ & - & $0.6 \cdot 10^{-2}$ & 0.99 \\
\hline \multirow{10}{*}{ Phased } & \multirow{2}{*}{$\mathrm{L}_{f}$} & set $A$ & - & $8 \pm 3$ & - & $1.1 \cdot 10^{-2}$ & 0.98 \\
\hline & & set B & - & $3 \pm 2$ & - & $-0.9 \cdot 10^{-2}$ & 0.98 \\
\hline & \multirow[b]{2}{*}{$\mathrm{LM}_{\mathrm{f}}$} & set $A$ & - & $17 \pm 4$ & - & $1.4 \cdot 10^{-2}$ & 0.99 \\
\hline & & set $B$ & - & $11 \pm 3$ & - & $-0.9 \cdot 10^{-2}$ & 0.99 \\
\hline & \multirow{2}{*}{$M_{f}$} & set $A$ & - & $14 \pm 4$ & - & $3.6 \cdot 10^{-3}$ & 0.99 \\
\hline & & set B & - & $13 \pm 4$ & - & $-1.8 \cdot 10^{-3}$ & 0.94 \\
\hline & \multirow{2}{*}{$\mathrm{MH}_{\mathrm{f}}$} & set $A$ & - & $8 \pm 3$ & - & $0.9 \cdot 10^{-2}$ & 0.99 \\
\hline & & set $B$ & - & $12 \pm 3$ & - & $0.5 \cdot 10^{-3}$ & 0.97 \\
\hline & \multirow[b]{2}{*}{$\mathrm{H}_{\mathrm{f}}$} & set $A$ & - & $10 \pm 3$ & - & $0.5 \cdot 10^{-2}$ & 0.99 \\
\hline & & set B & - & $5 \pm 2$ & - & $1.4 \cdot 10^{-3}$ & 0.99 \\
\hline
\end{tabular}

As regards the CIRS model 069 simulator, no low-frequency oscillation was detected through the phased array probe. In both linear and convex array probes, a high number of oscillations was detected in correspondence of the medium-high flow regime $\mathrm{MH}_{\mathrm{f}}$ (Figure 7). This could be due to a dampener failure in correspondence of such flow regime. Similarly to Gammex $1425 \mathrm{~A}$, a higher number of high velocity pulses was detected for both convex and phased array probes.

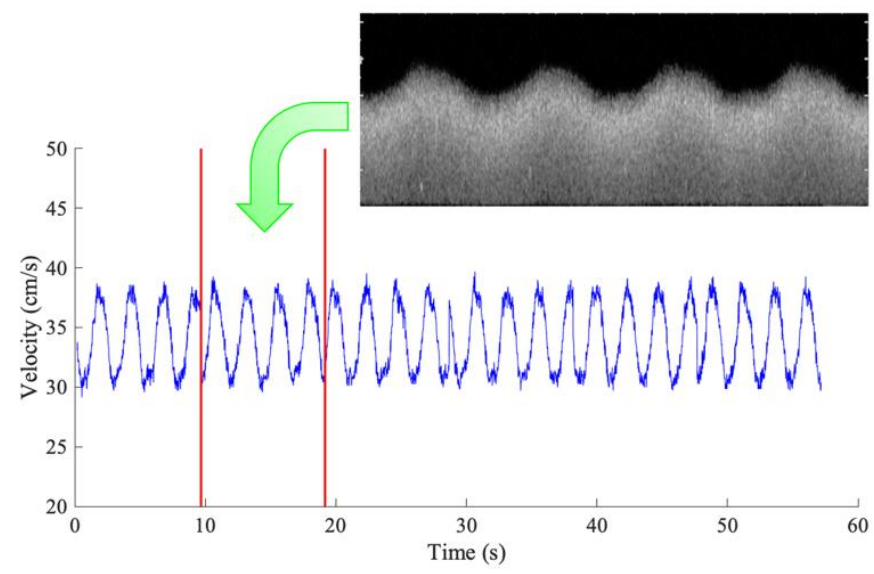

Figure 6. Example of the sinusoidal trend in $\mathrm{LM}_{\mathrm{f}}$ regime for Gammex 1425A acquired with the linear array probe in set $A$.
By comparing the two Doppler phantoms outcomes retrieved, Gammex 1425A shows the lowest number of lowfrequency oscillations (by excluding the particular case of $\mathrm{LM}_{\mathrm{f}}$ regime), when compared to both linear and convex array probes of the CIRS model 069, while for the phased array one no oscillations were detected. Conversely, Gammex 1425A globally shows the highest number of high velocity pulses compared to the CIRS model 069 for both linear and convex array probes, while such issue seems to be reversed for phased array probe.

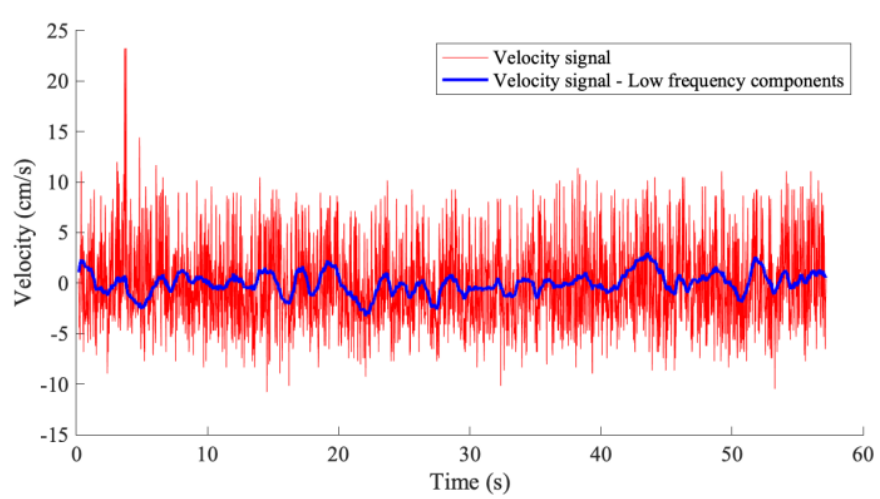

Figure 7. Example of the low frequency oscillations in $\mathrm{MH}_{\mathrm{f}}$ regime for $\mathrm{CIRS}$ model 069 acquired with the linear array probe in set $B$. 
Since the same number of PUT failures can have a different relevance depending on the intended use of the ultrasound system to be checked through the PUT (e.g., in echocardiography, obstetrics and gynecology, pediatrics, etc.), the EMoDICA-STFT may be applied with different thresholds by means of ad hoc US systems and probe models over time. Therefore, the same PUT may be suitable for testing a restricted number of US scanners only. This could be an advantage for the technical assessment of the above medical devices, as well as for PUT maintenance.

\section{CONCLUSIONS}

Doppler phantoms are standard reference test devices that, nowadays, are not yet included in a shared standard focusing on the objective evaluation of their performances and failures. In particular, phantom stability assessment is currently carried out through visual and subjective evaluations, or without a rigorous protocol. Therefore, in the present study, a novel method, named EMoDICA-STFT, based on the combined application of EMD, ICA and STFT techniques, is proposed and tested to automatically determine, through the processing of PW Doppler spectrograms, the number of phantom failures. The main flow phantom failures were classified as low frequency oscillations, high velocity pulses and velocity drifts. Data were collected from two flow phantoms by a single diagnostic US system equipped with three probe models. Tests were carried out in two different US configuration settings and five flow regimes set on the test objects. After a series of simulations, adaptive thresholds for the detection of each failure were determined depending on the standard deviation of the PUT flow velocity. Consequently, EMoDICA-STFT method was applied to the maximum flow velocity signals extracted from the PW Doppler spectrograms through an automatic processing. Finally, the number of detected failures was found for both Doppler phantoms. On the basis of the promising outcomes, further studies should be carried out (a) on a higher number of Doppler phantoms, (b) on a larger number of US diagnostic systems and (c) including an in-depth investigation of the proposed method uncertainty.

\section{ACKNOWLEDGEMENT}

The Authors wish to thank HITACHI Healthcare and Jan Galo of the Clinical Engineering Service at I.R.C.C.S. Children Hospital Bambino Gesù for hardware supply and technical assistance in data collection.

\section{REFERENCES}

[1] International Electrotechnical Commission, IEC 61685:2002-04, Ultrasonics - Flow measurement systems - Flow test object, 2002.

[2] J. E. Browne, A review of ultrasound quality assurance protocols and test devices, Phys. Med. 30 (2014) pp. 742-751. DOI: 10.1016/j.ejmp.2014.08.003

[3] S. M. Shalbi, A. A. Oglat, B. Albarbar, F. Elkut, M. A. Qaeed, A. A. Arra, A brief review for common Doppler ultrasound flow phantoms, J. Med. Ultrasound 28 (2020) pp. 138-142. DOI: $10.4103 /$ imu.jmu 9619

[4] K. K. Dakok, M. Z. Matjafri, N. Suardi, A. A. Oglat, S. E. Nabasu, A review of carotid artery phantoms for Doppler ultrasound applications, J. Med. Ultrasound 29 (2021) pp. 157-166. DOI: $10.4103 /$ imu.jimu 16420

[5] A. A. Oglat, M. Z. Matjafri, N. Suardi, M. A. Abdelrahman, M. A. Oqlat, A. A. Oqlat, A new scatter particle and mixture fluid for preparing blood mimicking fluid for wall-less flow phantom, J. Med. Ultrasound 26 (2018) pp. 134-142.
DOI: $10.4103 /$ imu.jmu $7 \quad 18$

[6] M. Alshipli, M. A. Sayah, A. A. Oglat, Compatibility and validation of a recent developed artificial blood through the vascular phantom using doppler ultrasound color- and motion-mode techniques, J. Med. Ultrasound 28 (2020) pp. 219-224.

DOI: $10.4103 /$ imu.jmu 11619

[7] A. A. Oglat, M. Z. Matjafri, N. Suardi, M. A. Oqlat, A. A. Oqlat, M. A. Abdelrahman, O. F. Farhat, M. S. Ahmad, B. N. Alkhateb, S. J. Gemanam, S. M. Shalbi, R. Abdalrheem, M. Shipli, M. Marashdeh, Characterization and construction of a robust and elastic wall-less flow phantom for high pressure flow rate using doppler ultrasound applications, Natural and Engineering Sciences 3 (2018) pp. 359-377.

DOI: $10.28978 /$ nesciences.468972

[8] G. Fiori, F. Fuiano, A. Scorza, J. Galo, S. Conforto, S. A. Sciuto, A preliminary study on the adaptive SNR threshold method for depth of penetration measurements in diagnostic ultrasounds, Appl. Sci. 10 (2020).

DOI: $10.3390 /$ app10186533

[9] A. Scorza, G. Lupi, S. A. Sciuto, F. Bini, F. Marinozzi, A novel approach to a phantom based method for maximum depth of penetration measurement in diagnostic ultrasound: a preliminary study, 2015 IEEE International Symposium on Medical Measurements and Applications (MeMeA), Turin, Italy, 7 - 9 May 2015.

DOI: $10.1109 / \mathrm{MeMeA} .2015 .7145230$

[10] G. Fiori, F. Fuiano, A. Scorza, J. Galo, S. Conforto, S. A. Sciuto, A preliminary study on an image analysis based method for lowest detectable signal measurements in Pulsed Wave Doppler ultrasounds, ACTA IMEKO 10 (2021) pp. 126-132.

DOI: $10.21014 /$ acta imeko.v10i2.1051

[11] G. Fiori, F. Fuiano, A. Scorza, M. Schmid, J. Galo, S. Conforto, S. A. Sciuto, A novel sensitivity index from the flow velocity variation in quality control for PW Doppler: a preliminary study, Proc. of 2021 IEEE International Symposium on Medical Measurements and Applications (MeMeA), Neuchâtel, Switzerland, 23 - 25 June 2021. DOI: $10.1109 / \mathrm{MeMeA} 52024.2021 .9478686$

[12] G. Fiori, A. Scorza, M. Schmid, J. Galo, S. Conforto, S. A. Sciuto, A preliminary study on the average maximum velocity sensitivity index from flow velocity variation in quality control for Color Doppler, Measurement: Sensors 18 (2021). DOI: $10.1016 /$ j.measen.2021.100245

[13] S. Cournane, A. J. Fagan, J. E. Browne, An audit of a hospitalbased Doppler ultrasound quality control protocol using a commercial string Doppler phantom, Phys. Med. 30 (2014) pp. 380-384.

DOI: $10.1016 /$ j.ejmp.2013.10.001

[14] IPEM Report No 102, Quality assurance of ultrasound imaging systems, 2010, ISBN 978-1-903613-43-6.

[15] K. V. Ramnarine, T. Anderson, P. R. Hoskins, Construction and geometric stability of physiological flow rate wall-less stenosis phantoms, Ultrasound Med. Biol. 27 (2001) pp. 245-250. DOI: $10.1016 / \mathrm{s} 0301-5629(00) 00304-5$

[16] A. Malone, D. Chari, S. Cournane, I. Naydenova, A. Fagan, J. Browne, Investigation of the assessment of low degree $(<50 \%)$ renal artery stenosis based on velocity flow profile analysis using Doppler ultrasound: an in-vitro study, Phys. Med. 65 (2019) pp. 209-218. DOI: $10.1016 /$ i.ejmp. 2019.08 .016

[17] J. V. Grice, D. R. Pickens, R. R. Price, Technical note: a new phantom design for routine testing of Doppler ultrasound, Med. Phys. 43 (2016) pp. 4431-4434. DOI: $\underline{10.1118 / 1.4954205}$

[18] M. Y. Park, S. E. Jung, J. Y. Byun, J. H. Kim, G. E. Joo, Effect of beam-flow angle on velocity measurements in modern Doppler ultrasound systems, AJR Am. J. Roentgenol. 198 (2012) pp. 11391143.

DOI: $\underline{10.2214 / \text { ajr.11.7475 }}$ 
[19] S. M. Patel, P. E. Allaire, H. G. Wood, A. L. Throckmorton, C. G Tribble, D. B. Olsen, Methods of failure and reliability assessment for mechanical heart pumps, Artif. Organs 29 (2005) pp. 15-25. DOI: $10.1111 / j .1525-1594.2004 .29006 . x$

[20] K. K. McKee, G. Forbes, I. Mazhar, R. Entwistle, I. Howard, A review of major centrifugal pump failure modes with application to the water supply and sewerage industries, Proc. of the ICOMS Asset Management Conference, Gold Coast, QLD, Australia, 16 May 2011. Online [Accessed 4 December 2021] https://espace.curtin.edu.au/bitstream/handle/20.500.11937/28 560/159989 38214 ICOMS $\% 20-\% 20$ Paper $\% 2032 \% 20$ $\% 20 \mathrm{~K} \% 20 \mathrm{McKee} \cdot \mathrm{pd}$ ? sequence $=2$ \&isAllowed $=\mathrm{y}$

[21] G. Fiori, F. Fuiano, A. Scorza, M. Schmid, J. Galo, S. Conforto, S A. Sciuto, Doppler flow phantom stability assessment through STFT technique in medical PW Doppler: a preliminary study, Proc. of 2021 IEEE International Workshop on Metrology for Industry 4.0 \& IoT (MetroInd4.0\&IoT), Rome, Italy, 7 - 9 June 2021.

DOI: 10.1109/MetroInd4.0IoT51437.2021.9488513

[22] B. Mijović, M. De Vos, I. Gligorijević, J. Taelman, S. Van Huffel, Source separation from single-channel recordings by combining empirical-mode decomposition and independent component analysis, IEEE Trans. Biomed. Eng. 57 (2010) pp. 2188-2196. DOI: $10.1109 /$ TBME.2010.2051440

[23] Z. Wu, N. E. Huang, Ensemble empirical mode decomposition: a noise-assisted data analysis method, Adv. Adaptive Data Anal. 1 (2009) pp. 1-41. DOI: $10.1142 / \mathrm{S} 1793536909000047$

[24] N. E. Huang, S. Zheng, S. R. Long, M. C. Wu, H. H. Shih, Q. Zheng, N.-C. Yen, C. C. Tung, H. H. Liu, The empirical mode decomposition and the Hilbert spectrum for nonlinear and nonstationary time series analysis, Proc. R. Soc. Lond. A. 454 (1998) pp. 903-995.

DOI: $\underline{10.1098 / \mathrm{rspa} .1998 .0193}$

[25] G. R. Naik, S. E. Selvan, H. T. Nguyen, Single-channel EMG classification with ensemble-empirical-mode-decompositionbased ICA for diagnosing neuromuscular disorders, IEEE Trans. Neural Syst. Rehabil. Eng. 24 (2016) pp. 734-743.

DOI: $\underline{10.1109 / \text { tnsre.2015.2454503 }}$

[26] G. Wang, C. Teng, K. Li, Z. Zhang, X. Yan, The removal of EOG artifacts from EEG signals using independent component analysis and multivariate empirical mode decomposition, IEEE J. Biomed. Health Inform. 20 (2016) pp. 1301-1308. DOI: $\underline{10.1109 / \text { ibhi.2015.2450196 }}$

[27] H. R. Ahmadi, N. Mahdavi, M. Bayat, A novel damage identification method based on short time Fourier transform and a new efficient index, Structures 33 (2021) pp. 3605-3614. DOI: $10.1016 / \mathrm{j}$.istruc. 2021.06 .081

[28] A. Khan, D.-K. Ko, S. C. Lim, H. S. Kim, Structural vibrationbased classification and prediction of delamination in smart composite laminates using deep learning neural network, Composites Part B: Engineering 161 (2019) pp. 586-594. DOI: 10.1016/i.compositesb.2018.12.118
[29] M. Le, J. Kim, S. Kim, J. Lee, B-scan ultrasonic testing of rivets in multilayer structures based on short-time Fourier transform analysis, Measurement 128 (2018) pp. 495-503. DOI: $10.1016 / j . m e a s u r e m e n t .2018 .06 .049$

[30] D. Cordes, M. F. Kaleem, Z. Yang, X. Zhuang, T. Curran, K. R. Sreenivasan, V. R. Mishra, R. Nandy, R. R. Walsh, Energy-period profiles of brain networks in group fMRI resting-state data: a comparison of empirical mode decomposition with the short-time Fourier transform and the discrete wavelet transform, Front. Neurosci. 15 (2021).

DOI: $10.3389 /$ fnins.2021.663403

[31] V. Gupta, M. Mittal, QRS complex detection using STFT, chaos analysis, and PCA in standard and real-time ECG databases, J. Inst. Eng. India Ser. B 100 (2019) pp. 489-497.

DOI: $\underline{10.1007 / \mathrm{s} 40031-019-00398-9}$

[32] A.-C. Tsai, J.-J. Luh, T.-T. Lin, A novel STFT-ranking feature of multi-channel EMG for motion pattern recognition, Expert Systems with Applications 42 (2015) pp. 3327-3341. DOI: $10.1016 /$ i.eswa.2014.11.044

[33] A. Hyvärinen, P. Ramkumar, L. Parkkonen, R. Hari, Independent component analysis of short-time Fourier transforms for spontaneous EEG/MEG analysis, NeuroImage 49 (2010) pp. 257-271.

DOI: $10.1016 /$ j.neuroimage.2009.08.028

[34] A. Hyvärinen, J. Karhunen, E. Oja, What is independent component analysis, in: Independent Component Analysis. A. Hyvärinen, J. Karhunen, E. Oja (editors). Wiley-Interscience, New York, NY, USA, 2001, ISBN 0-471-22131-7, pp. 147-163.

[35] A. Hyvärinen, E. Oja, A fast fixed-point algorithm for independent component analysis, Neural Computation 9 (1997) pp. 1483-1492. DOI: $\underline{10.1162 / \text { neco.1997.9.7.1483 }}$

[36] http://research.ics.aalto.fi/ica/software.shtml Online [Accessed 4 December 2021]

[37] B. Boashash, Heuristic formulation of time-frequency distributions, in: Time-frequency signal analysis and processing. B. Boashash (editor). Academic Press, 2015, ISBN 978-0-12-3984999, pp. 65-102.

[38] Gammex, Optimazer 1425A: Ultrasound image analyzer for Doppler and gray scale scanners. Online [Accessed 4 December 2021] https://cspmedical.com/content/1021086 doppler user manual.pdf

[39] CIRS Tissue Simulation \& Phantom Technology, Doppler ultrasound flow simulator - model 069. Online [Accessed 4 December 2021] http://www.3000buy.com/uploads/soft/180118/Cirs069.pdf

[40] F. Marinozzi, F. Bini, A. D’Orazio, A. Scorza, Performance tests of sonographic instruments for the measure of flow speed, 2008 IEEE International Workshop on Imaging Systems and Techniques (IST), Chania, Greece, 10 - 12 September 2008. DOI: $10.1109 /$ IST.2008.4659939

[41] J. R. Taylor, An introduction to error analysis: the study of uncertainties in physical measurements, University Science Books, Sausalito, CA, USA, 1997, ISBN 0-935702-75-X, pp. 245-260. 